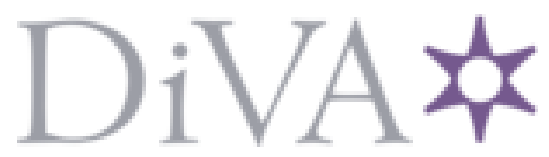

http://www.diva-portal.org

This is the published version of a paper published in Journal of research on adolescence.

Citation for the original published paper (version of record):

Veenstra, R., Dijkstra, J., Steglich, C., Van Zalk, M. (2013)

Network-Behavior Dynamics.

Journal of research on adolescence, 23: 399-412

http://dx.doi.org/10.1111/jora.12070

Access to the published version may require subscription.

N.B. When citing this work, cite the original published paper.

Permanent link to this version:

http://urn.kb.se/resolve?urn=urn:nbn:se:oru:diva-43429 


\section{Network-Behavior Dynamics}

\author{
René Veenstra, Jan Kornelis Dijkstra, and \\ Christian Steglich \\ University of Groningen
}

\author{
Maarten H. W. Van Zalk \\ Örebro University and Utrecht University
}

Researchers have become increasingly interested in disentangling selection and influence processes. This literature review provides context for the special issue on network-behavior dynamics. It brings together important conceptual, methodological, and empirical contributions focusing on longitudinal social network modeling. First, an overview of mechanisms underlying selection and influence is given. After a description of the shortcomings of previous studies in this area, the stochastic actor-based model is sketched; this is used in this special issue to examine network-behavior dynamics. The preconditions for such analyses are discussed, as are common model specification issues. Next, recent empirical advances in research on adolescence are discussed, focusing on new insights into moderating effects, initiation of behaviors, time heterogeneity, mediation effects, and negative ties.

Relationships with peers provide an important context for social development and adjustment. Two fundamental processes underlying network-behavior dynamics are key to our understanding of adolescents' development: selection and influence. Selection processes concern, for example, whom adolescents choose to hang out or be friends with. They affect the formation and dissolution of relationships. One important and well-known class of selection processes is based on similarity. Beginning in early childhood, children tend to sort themselves nonrandomly into friendships, selecting peers who are more or less similar to themselves. However, similarity is not the only possible basis of relationships. Selection processes refer more generally to any mechanism by which individuals adjust their relationships in response to the social

\footnotetext{
We would like to thank all reviewers for their valuable comments on earlier drafts of the contributions to this special issue. All articles in this special issue were discussed during weekly meetings of the research line Social Development of Adolescents at the University of Groningen. This research line is coordinated by René Veenstra and Jan Kornelis Dijkstra. We thank the following members for their useful inputs: Anke Munniksma, Ashwin Rambaran, Beau Oldenburg, Britta Ruschoff, David Duijst, Femke Munniksma, Gijs Huitsing, Jelle Sijtsema, Judit Pál, Kim Pattiselanno, Loes van Rijsewijk, Louisa Firnenburg, Miranda Sentse, Rozemarijn van der Ploeg, Tina Kretschmer, Vera Hanewinkel, and Vera Heininga. Part of this research was funded by the Netherlands Organisation for Scientific Research (NWO): VENI Project number 451-10-012 awarded to Jan Kornelis Dijkstra (2010).

Requests for reprints should be sent to René Veenstra, Department of Sociology and Interuniversity Center for Social Science Theory and Methodology (ICS), University of Groningen, Grote Rozenstraat 31, 9712 TG, Groningen, The Netherlands. E-mail: d.r.veenstra@rug.nl
}

context, their own behaviors, and their peers' behaviors.

In contrast, peer relationships also shape individual behaviors and other changeable characteristics (e.g., attitudes and opinions). Whom adolescents hang out with and whom they consider to be friends affects their individual development. Influence processes refer more generally to individuals changing their behavior or attitudes in response to (the behavior or attitudes of) the peers they affiliate with.

A methodological challenge here is that selection and influence processes might both result in the same empirical phenomenon: similarity of connected individuals. This similarity may result from similar individuals choosing each other (selection), which suggests that behavior remains similar, but relationships change, or from connected individuals becoming increasingly similar (influence), which suggests that relationships remain stable but behavior changes. This shows, first, that longitudinal analysis is necessary if one aims to assess selection and influence processes. Second, the sequence of changes in the network and the behavior represents the mutual dependence between network dynamics and behavior dynamics. It is, therefore, necessary to examine behavior and network dynamics simultaneously, using a method that is capable of accounting for this simultaneity.

While developmental researchers have long understood that the study of influence requires consideration of selection and that the study of

(C) 2013 The Authors

Journal of Research on Adolescence (C) 2013 Society for Research on Adolescence DOI: $10.1111 /$ jora. 12070 
selection requires consideration of influence (see also Veenstra \& Dijkstra, 2011), they were not immediately successful in disentangling the two processes and assessing the relative contribution of selection and influence to observed similarity in peer relationships in a statistically sound way. This became possible with advances in longitudinal social network analysis using stochastic actor-based modeling (Snijders, Steglich, \& Schweinberger, 2007). This method requires longitudinal social network data with information about all individuals in a meaningfully delineated social group and their changes in relationships and behaviors traced over two or more observation moments.

Although selection based on similarity and influence that results in similarity is the primary interest of much of the research reported in this special issue, other selection and influence processes can also be investigated using the stochastic actorbased model: for example, whether adolescents have preferences for peers with particular behaviors or whether social influence results in individuals with particular network positions being more likely to adopt certain behaviors.

The articles in this special issue focus on complete social networks, where relationships among a bounded set of individuals are measured as well as individuals' attributes or behaviors. A social network is seen as the relationships among a collection of individuals, with overall structural properties (e.g., the density of relationships among all individuals in the network) and structural properties of individuals located in the network (e.g., if individuals are popular or isolated). These properties can change over time.

\section{THEORETICAL OVERVIEW}

\section{Selection and Influence Processes}

The idea that humans select their friends based on similarity can be traced back at least to Greek antiquity (McPherson, Smith-Lovin, \& Cook, 2001). Lazarsfeld and Merton (1954) coined the term homophily for this process; Byrne (1971) referred to it as similarity attraction. People select similar others as friends because, on average, those who are similar in behaviors, characteristics, and attitudes understand each other better. Similarity increases trustworthiness and predictability, enabling individuals to communicate with less effort and with shared feelings of understanding and belongingness, which makes these relationships more rewarding and stable and reduces conflicts. In addition to providing a basis for mutual approval, shared characteristics provide a source of validation for development and reinforcement of social identity (Hallinan, 1980).

Peer selection is not only steered by preferences, but also depends on the composition of the pool of peers, which structures and restricts choices (Blau, 1977). For instance, the student body within a school tends to have much more in common, such as socioeconomic status, ethnicity, or intelligence, than do individuals in society at large. As schools tend to be homogeneous in composition, the chances of meeting and affiliating with similar peers are high. Feld (1982) has noted that activity foci, social settings that structure people's actions and interactions, increase the likelihood of friendship with similar others. Similarity of friends may, therefore, be the largely unintended result of the greater or lesser opportunity to meet similar others in one's daily life (see also Osgood \& Anderson, 2004).

Opportunities for affiliation also have an impact on influence processes in which individuals adopt behaviors and attitudes of their peers in the network, resulting in peer group homogeneity (Cohen, 1977; Friedkin, 1998). The importance of relationships as socializing agents can be traced back to Durkheim (1897), who argued that all types of behavior are influenced by social norms and that norm conformity is enforced through membership and integration in social groups. Influence processes might work via mechanisms of social learning and imitation (Bandura, 1977), expected rewards of conformity (Burgess \& Akers, 1966), social facilitation (Zajonc, 1968), or peer pressure (Warr, 2002). In sum, similarity of friends may be a result not just of selection processes or of opportunity structures, but also of influence processes, referring to the tendency of individuals to become more similar to one another in response to the behaviors or attitudes of others they are connected to in their network.

\section{Shortcomings of Previous Research on Selection and Influence}

Studies have documented substantial similarity between adolescents' behavioral repertoires and those of their friends. Until the recent development of statistical models to examine coevolving network and behavior processes, four primary limitations restricted the ability to make firm statements about the underlying processes responsible for similarity among friends (Steglich, Snijders, \& Pearson, 2010). 
First, assessments of peer characteristics often came from the focal respondent (referring to that respondent's reports on characteristics of his or her friends), a strategy that potentially inflates the magnitude of peer effects owing to exaggeration of similarity to friends in behavior, an assumed similarity or false consensus bias.

Second, changes in behavior and relationships that occurred in between observation moments were not modeled in previous models. However, a true test of selection and influence effects should take such unobserved changes into account to avoid overestimation (see for an illustration Veenstra \& Steglich, 2012).

A third limitation of previous models is their exclusive focus on dyadic relationships and thus a failure to control for the effect of the network structure on network-behavior dynamics. Several structural selection processes are known to play a role. For example, friendships are more likely to be established when persons share a common friend (Davis, 1970), called a transitivity effect. Such a transitivity effect (rather than similarity in behavior) might account for friendship formation between network members. Thus, when friendship formation between two smoking adolescents occurs via a shared friend (transitivity), leaving out an estimate for transitivity would cause one to incorrectly attribute it to their smoking behavior. Not controlling for structural network tendencies can lead to overestimation of selection effects, which, in turn, affects estimates for influence through failure to rule out selection effects in a statistically sound way (Steglich et al., 2010).

A final limitation is the failure to examine complete networks. To identify the determinants of selection, it is not sufficient to know who was selected as network partner; it is also necessary to know who was not selected. Likewise, for identifying peer influence effects, it is necessary to know what the absence of influence looks like, referring to how behavior changes in the absence of relationships and opportunities for influence. In earlier studies, this was typically acknowledged by comparing a sample of dyads in which the individuals were (and stayed) socially connected to each other in the network with a matched sample of dyads in which the individuals were (and stayed) unconnected. However, use of the crucially important control for selection processes is impossible in this approach (Veenstra \& Steglich, 2012).

To overcome these shortcomings and unravel selection and influence processes, researchers should incorporate data about the presence and absence of relationships among a set of individuals as well as the behavior of these individuals. This approach explicitly samples individuals who are socially connected and typically represent some naturally bounded group (e.g., children in a school). Many dependencies arise in the social network. It is not warranted to apply many commonly used statistical procedures, but it is necessary to apply procedures in which network dependencies are part of the modeling. The stochastic actor-based model was developed to model network evolution and behavior change as two interdependent processes (Steglich et al., 2010). The model is implemented in the Simulation Investigation for Empirical Network Analyses (SIENA) software and is freely available as R-package: http://www.stats.ox. ac.uk/ snijders/siena.

\section{STOCHASTIC ACTOR-BASED MODEL}

As prerequisites for stochastic actor-based modeling, we assume that the empirical data consist of a sequence of at least two observations of a network and a behavioral variable, measured at time points $t_{1}, t_{2}, \ldots, t_{m}$ on a given meaningfully delineated set of actors such as students in the same school class. Ties are the bilateral constituents of relationships, directed from one actor to another. In principle, ties are under the control of the sending actor. The tie variables are binary, denoted by $x_{i j}$. A tie from actor $i$ to actor $j$, denoted $i \rightarrow j$, is either present or absent ( $x_{i j}$ then having values 1 and 0 , respectively). Tie variables constitute the network, represented by its $n \times n$ adjacency matrix $x=\left(x_{i j}\right)$ (self-ties are excluded), where $n$ is the total number of actors. Co-dependent behavior variables are assumed to be discrete and ordinal and represented by a vector $z=\left(z_{i}\right)$.

Figure 1 illustrates the correspondence between a network-behavior configuration and its representation as an adjacency matrix and a behavior vector. The dependent variable in an actor-based analysis is the time series of observed matrices and behavior vectors, $(x, z)\left(t_{1}\right),(x, z)\left(t_{2}\right), \ldots,(x, z)\left(t_{m}\right)$.

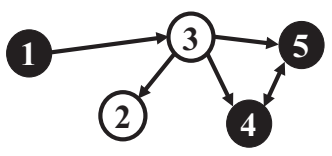

$$
\boldsymbol{x}=\left(\begin{array}{lllll}
0 & 0 & 1 & 0 & 0 \\
0 & 0 & 0 & 0 & 0 \\
0 & 1 & 0 & 1 & 1 \\
0 & 0 & 0 & 0 & 1 \\
0 & 0 & 0 & 1 & 0
\end{array}\right), \quad z=\left(\begin{array}{l}
1 \\
0 \\
0 \\
1 \\
1
\end{array}\right)
$$

FIGURE 1 Scheme of a binary social network with binary behavioral variable (color) and its notation as adjacency matrix $x$ and behavior vector $z$. 
The actor-based model interprets this discrete time series of data as the cumulative result of an unobserved sequence of elementary changes, resulting from decisions taken by the actors between observation moments. As such, the approach overcomes the limitation that the underlying change process has not been observed and can only be inferred by modeling. To do so with some statistical power, two assumptions are made.

The first assumption concerns decomposability into the smallest possible changes. The unobserved change process is assumed to take place as gradually as possible, by "microsteps" that consist of either a change in a tie or a step up or down on the behavior scale. This enforces gradual change for a single actor.

The second assumption is that of conditional sequential independence. It is assumed that at any moment in time, the current network and the behavior configuration are sufficiently informative, meaning that the history of the evolution process is fully mediated by the current state (the Markov assumption). This implies that some potentially important quantities (like the age of a network link or whether a behavior level was approached from higher or from lower levels) are not included in the model.

By enforcing a sequential nature on the decisions that accumulate to the observed changes, parameter estimation is facilitated (Holland \& Leinhardt, 1977) and the interdependence of ties and individual behavior typical of network data is enacted through feedback processes in continuous time. Due to the unobserved nature of the exact sequence of changes connecting observation moments, simulation-based inference becomes necessary for estimating model parameters (Snijders, Van de Bunt, \& Steglich, 2010).

The basic network-behavior dynamics model has four parts: two rate functions (expressing the amount of change) and two objective functions (expressing the direction of change), one each for decisions about network change and behavior change. Any imputed sequence amounts to the repeated identification of a focal actor (ego) who gets the opportunity to make a change to his or her tie or behavior (or to make no change), and the identification of the change outcome. The identification of ego is modeled using the so-called rate functions $\lambda$, which indicates the frequency of opportunities for behavior and network changes. These functions jointly parameterize an exponential distribution of waiting times. Both network and behavior rate functions can depend on individual characteristics of the actors, including features of their network position.

We will not elaborate further on the rate functions because in the school-centered friendship context addressed in this special issue, it seems reasonable to assume equal numbers of change opportunities and hence use a period-wise constant rate function for all individuals. The only exceptions to this homogeneity assumption are the articles by Light, Greenan, Rusby, Nies, and Snijders (2013) and De la Haye, Green, Kennedy, Pollard, and Tucker (2013), who model behavior initiation (rather than behavior change on a scale), which requires more nuanced rate function models.

Bounded by the selected combination of the focal actor (ego) and decision domain (network or behavior), ego may change one outgoing tie (either create or break a tie), change their own behavior (going one step up or down on the behavior scale), or do nothing (keep the status quo). The outcome probabilities of these decisions depend on the objective functions $f$ for behavior and network changes. Objective functions parameterize a multinomial logit choice model. They indicate how attractive it is for an actor to go from an old to a new state. These functions summarize the information actors use to adjust their position in the network and their position on the behavior scale. Potential components of this function can be structure-based (endogenous) effects, such as the tendency to form reciprocal relationships, or attribute-based (exogenous) effects, such as the preference for associating with similar others. It is assumed that actors have full information about network structure and composition and can accurately assess the consequences of their decisions. Based on their evaluations of these consequences, actors make changes that will maximize their objective functions, subject to the constraints of network structure, behavior of all actors in the network, changes made by others in the network, and random influences (Snijders et al., 2010).

\section{PRECONDITIONS FOR AN ANALYSIS}

Prior to applying the stochastic actor-based model, several features of the data should be evaluated. First, the Moran's network autocorrelation coefficient can be used to determine the presence of an association between the network and a behavioral variable, justifying the use of a model to explain this association with selection and influence effects. Moran's network autocorrelation coefficient uses the correlation in the behavior variable between 
pairs of individuals in the network and the degree to which network members who share a relationship deviate from the average in the network. It is high when friends are in a similar way either positively or negatively different from the average score. As with other correlation coefficients, it can range from -1 (perfect dissimilarity) through 0 (perfect independence) to +1 (perfect similarity).

Second, a researcher should determine whether the data are sufficiently informative to allow for identification of effects. To get a rough impression of the statistical power of the data, it is possible to examine the amount of change and stability in the network and the behavior. In general, the total number of changes between consecutive observations should be large enough to provide information for estimating parameters. More changes will give more information and, thereby, allow the fitting of more sophisticated models. However, a complete turnover of friendship nominations would call into question the assumption that the waves are consecutive observations of a gradually changing network. If the stable part of the network is small, the consecutive observations are probably too far apart. This implies that, when designing a study, the researcher needs to have a reasonable estimate of the expected change. For instance, to investigate the development of a friendship network among a group of initially mutual strangers (e.g., freshmen in high school), it may be advisable to plan the observation moments to be separated by only a few weeks in the beginning and to enlarge the period between observations after a couple of months.

The Hamming distance can be used to determine the total number of friendship nominations in the network for which there is observed change between data observations and includes the sum of new nominations (friends at the end of the period, but not at the beginning) and lost nominations (vice versa). It is a lower boundary for the number of microsteps needed to get from one network observation to the next, and as such gives an indication of the statistical power for identifying parameters for network dynamics.

The Jaccard index is the fraction of stable friendship nominations among the new, lost, and stable ties between observed data points (it disregards stable absence of friendship). Without a good fraction of stability in the data, an initial measurement of the network is uninformative for the evolution process toward the next measurement, and the two measurement points might better be analyzed separately. Based on experience with the method, Jaccard indices of around $30 \%$ indicate sufficient stability of classroom-sized networks; for larger networks, also lower indices $(20 \%-25 \%)$ can be tolerated (see Simpkins, Schaefer, Price, \& Vest, 2013).

Finally, it is also important to examine stability and change in behavior, looking at the fraction of respondents who changed and the direction and amount of this change from one observation to the next, and over the whole observation period. Because behavior data are fewer than network data (vector vs. matrix), the behavior dynamics specification should be considerably more parsimonious than the network dynamics specification.

\section{MODEL SPECIFICATION}

Network dynamics, meaning changes in the structure of the network and thus ties, are explained by (a) structural network effects and (b) attributerelated selection effects. Social network research has found strong evidence that ties in the network are not independent of one another, but that the presence of ties and particular configurations of ties are likely to influence local changes in other ties. Thus, there are some clear tendencies toward dependence between ties. Such structural network effects are controlled for to avoid bias in other effects (see Figure 2).

Second, selection effects reflect changes in the peer network that depend on either relational (e.g., the geographical distance between two adolescents' homes) or individual attributes (e.g., sex). The latter can be differentiated into effects of preexisting similarities in behavior (selection similarity effect) and effects of behaviors on incoming ties (alter effect) and outgoing ties (ego effect).

Behavior dynamics may be captured by (c) behavioral tendencies and (d) influence effects. Behavioral tendencies are taken into account to model the distribution and baseline likelihood of changes in the behavior under investigation (including polarization or regression to the mean). Influence effects can include friends' influence as well as the influences of individuals' attributes on changes to their behavior (see Figure 2).

\section{Network Dynamics}

The following section discusses the structural network effects used in this special issue. For other possibilities, see the RSiena manual.

Outdegree (density) (i) $\longrightarrow$ (i). The outdegree effect corresponds to the number of friends an actor nominates. It is an intercept effect that reflects 


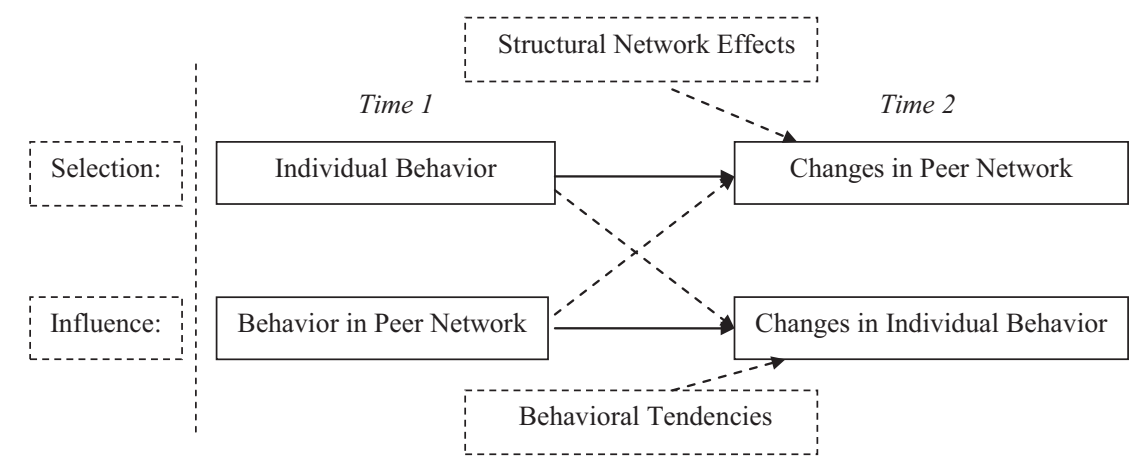

FIGURE 2 Representation of selection and influence effects. Note. The solid lines in figure express that individuals can change their peer network (selection) and behavior (influence) between two time points. The dashed lines express that selection and influence effects are estimated while the network structure and behavior at Time 1, the structural network effects (e.g., reciprocity, transitivity), and behavioral tendencies are taken into account.

the basic tendency to name friends at all and as such models the density of the network. Because the objective function parameters refer to a logistic scale and all articles in this issue reported densities of much less than $50 \%$, we always see a negative estimate for this parameter.

Reciprocity (i) $\longleftrightarrow$ (j). Another basic effect is the tendency toward reciprocation of choices, referring to mutual ties: $i$ chooses $j$ and $j$ chooses $i$. The parameter is zero when the (conditional) probability of reciprocation is identical to the (unconditional) probability of any tie existing. Results for all friendship networks in this issue show significant positive evidence for reciprocity, which is no surprise because friendship tends to be governed by the norms of reciprocation.

Triad structure. Effects incorporating structural information involving three or more actors are a crucial advantage of social network analysis over purely dyad-based approaches.

\section{a. Transitivity}

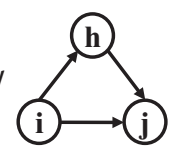

An essential feature of peer group structure is the tendency toward transitivity or transitive closure. Friends of friends become friends. The most straightforward formulation to operationalize transitivity is the transitive triplet statistics. This effect measures transitivity for an actor $i$ by counting the number of configurations $(i, h, j)$ in which all three ties $i \rightarrow h, h$ $\rightarrow j, i \rightarrow j$ are present (irrespective of whether there are also other ties between these three actors). The tie $i \rightarrow j$ becomes increasingly likely the more indirect connections (two paths) $i \rightarrow h \rightarrow j$ there are.

Another way of measuring transitivity is the transitive ties effect. It measures transitivity for actor $i$ by counting the number of other actors $j$ for which there is at least one intermediary $h$ to form a transitive triplet. A positive effect of transitive triplets or transitive ties was obtained in all studies on friendship dynamics reported in this issue.

A third and often-used statistic expressing transitivity is the tendency for actors to keep others at a sociometric distance of two, thus avoiding friendship with friends of friends. This measure is the converse of transitive closure. For that reason, a negative effect was obtained in all studies reported in this issue that estimated this parameter.

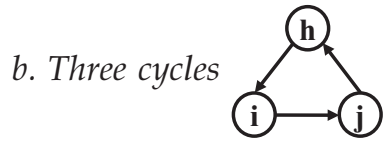

This measure represents the number of three cycles $i$ $\rightarrow j \rightarrow h \rightarrow i$ which actor $i$ is involved in. Three cycles are nonhierarchical, and a positive three-cycle effect can be interpreted as a tendency toward generalized exchange (Bearman, 1997). In many networks, there is a tendency to have a hierarchical ordering with relatively few three cycles. For that reason, studies in this issue found a negative estimate for the three-cycle parameter.

\section{c. Balance/structural equivalence}

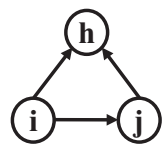

An effect closely related to transitivity is balance, which is the tendency to have ties with others who make similar network choices (Cartwright \& Harary, 1956). Intransitive relationships - where friends' evaluations of third parties diverge - are likely to create emotional tensions, making them unstable and leading individuals to change their perceptions or relationships in order to restore balance. The extent to which two actors make the same choices can be expressed simply as the number of outgoing 
choices and nonchoices that they have in common. Balance, thus, represents similarity between the outgoing ties of actor $i$ and the outgoing ties of the other actors $j$ to whom $i$ is tied. Some studies in this issue modeled this effect and found a positive estimate.

Degree distribution. Indegrees and outdegrees are primary characteristics of an actor's position in a network. The indegree is the number of ties directed at an actor, referring to the number of nominations received. The outdegree is the number of ties going from an actor, referring to the number of nominations given.

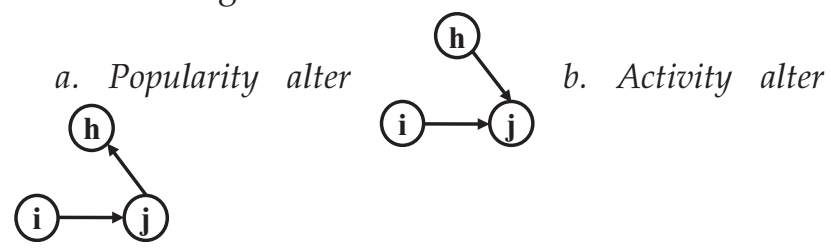

The popularity alter effect models the tendency to form ties with actors who have high indegrees already, referring to a "Matthew" effect (Merton, 1968) or preferential attachment, that is, increased attractiveness of already highly attractive individuals. In this issue, Simpkins et al. (2013) and Osgood et al. (2013) found a positive estimate for this effect. The activity alter effect models the tendency to form ties with actors who already have high outdegrees. In this issue, De la Haye, Green, et al. (2013) found a negative estimate for this effect, indicating that students avoided friendships with peers who nominated a large number of friends.

Covariates: Actor attribute effects. For an actor attribute, there are two basic effects: the ego (sender) effect $\langle\hat{i} \longrightarrow$ (i), measuring whether actors with higher attribute values (depicted by the diamond shape) tend to nominate more friends and hence have a higher outdegree, and the alter (receiver) effect $(\mathrm{i} \longrightarrow\langle\hat{\mathrm{j}}\rangle$, measuring whether actors with higher attribute values tend to be nominated more often and hence have higher indegrees. Actor covariates may be constant like sex or ethnicity, or subject to change like attitudes or behaviors.

Next, there are effects combining information on sender and receiver, illustrated as $\langle\hat{\mathrm{i}} \longrightarrow\langle\mathrm{i}\rangle$. The selection similarity effect measures whether ties tend to occur more often between actors with similar values on an attribute. This effect can be modeled for categorical or binary data and tests if actors prefer to have ties with network members who have the same value on the attribute. The Ego $\times$ Alter interaction also combines information on sender and receiver. A positive effect of that interaction reflects that individuals who have a high score on an attribute show a higher preference for friends who also have a high score on that attribute.

\section{Behavior Dynamics}

Behavioral tendencies: Linear and quadratic shape effects. Basic distributional features of the behavior variable are expressed using two shape parameters that should be estimated as control variables. The linear shape effect is an intercept expressing the average tendency toward low values (negative value) or high values (positive value) on the variables under investigation. The quadratic shape effect models the feedback effect of the behavior on itself (Snijders et al., 2010). A negative value indicates that behavior of respondents tends to regress to the mean (self-correcting mechanism). A positive value indicates that behavior of respondents tends to regress to the extremes of the scale (polarization or self-reinforcing mechanism). A self-correcting mechanism has been found in studies on, for example, weight status, junk food intake, physical activity, and victimization. A selfreinforcing mechanism has been found in the vast majority of studies on dynamics in delinquency, weapon carrying, and substance use. In the special case when the behavior variable is dichotomous, the quadratic shape effect is collinear with the linear shape effect and must not be included in the model.

Influence effects. The actor-based model can represent social influence in various ways. First, through the average similarity effect, expressing the preference of actors to be similar in behavior to their alters, in such a way that the total influence of alters is the same regardless of the number of alters an ego is directly connected to (referring to ego's outdegree). Second, through the total similarity effect, expressing the preference of actors to be similar in behavior to their alters, in such a way that the total influence of the alters is proportional to the number of alters. Third, through the average alter effect, expressing that actors whose alters have a higher average value of the behavior, also have themselves a stronger tendency toward high values on the behavior. The choice between these different representations of social influence has to be made on theoretical grounds. The average similarity effect is in line with theories about group norms; the average alter effect is in line with contagion 
theory. In the absence of specific theory, parameter choice can be facilitated by comparing different model specifications with a score type test (Snijders et al., 2007).

Network position can also have an effect on behavior dynamics. A positive effect of the actor's indegree and outdegree may indicate that those who are more active (higher outdegree) and popular (higher indegree) have a stronger tendency to display higher values of the behavior over time; see, for example, Osgood et al. (2013).

For actor covariates, a main effect of the covariates on behavior dynamics can be included, representing the influence of a covariate on behavior changes. In addition, it is possible that actor covariates moderate selection and influence effects, leading to an interaction between the covariate and selection or influence effects. We give examples of such moderating effects below.

\section{DYNAMICS IN NETWORKS AND A RANGE OF BEHAVIORS}

Theories on development in adolescence emphasize the importance of peers (and friendships in particular) as socializing agents for behavioral development. In the studies reported in this issue, researchers examined dynamics in networks and a range of behaviors, including externalizing problems, internalizing problems, substance use, healthcompromising behaviors, prosocial behavior, social goals, and happiness. Other topics recently investigated but not included in this issue are dynamics in networks and victimization (Sentse, Dijkstra, Salmivalli, \& Cillessen, 2013; Sijtsema, Rambaran, \& Ojanen, 2013), xenophobia (Van Zalk, Kerr, Van Zalk, \& Stattin, 2013), political behaviors (Dahl \& Van Zalk, 2014), and religious service attendance (Cheadle \& Schwadek, 2012).

\section{Externalizing Problems}

Selection and influence processes have also been investigated with respect to externalizing problems such as aggression and delinquency. In this issue, Logis, Rodkin, Gest, and Ahn (2013), Molano, Jones, Brown, and Aber (2013), and Rulison, Gest, and Loken (2013) show that ego's aggression is influenced by the aggression of their friends (see also Dijkstra, Berger, \& Lindenberg, 2011; Sijtsema et al., 2010). In previous studies, influence processes have also been investigated with respect to delinquency. These studies revealed that the influence effect on delinquency was found in studies that looked at the effects of peers in the same grade or town, and not of classmates (Dijkstra, Gest, Lindenberg, Veenstra, \& Cillessen, 2012; Svensson, Burk, Stattin, \& Kerr, 2012; Weerman, 2011). This suggests that peer influences leading to similar rates of antisocial behavior may occur through an active process in which antisocial behavior is reinforced by out-of-class friends.

Logis et al. (2013), Molano et al. (2013), and Rulison et al. (2013) provide no evidence for selection similarity in aggression. However, selection similarity has been found for delinquency (Burk, Van der Vorst, Kerr, \& Stattin, 2012; Kiuru, Burk, Laursen, Salmela-Aro, \& Nurmi, 2010; Knecht, Snijders, Baerveldt, Steglich, \& Raub, 2010). These studies describe the operation of peer selection processes through attraction for children who display a similar amount of antisocial behavior. In addition, these researchers have found that antisocial adolescents were nominated by others as friends and were more restrictive than others in the number of friendships that they mentioned (see also Dijkstra, Cillessen, \& Borch, 2013; Dijkstra et al., 2010). The combination of high indegree and low outdegree is indicative of a high social status.

\section{Internalizing Problems}

Selection and influence processes have also been investigated with respect to internalizing problems such as anxiety, depression, and loneliness. In a test of similarity attraction, Mercer and DeRosier (2010) reported that lonely children were more likely to select other lonely children as friends. The same was found for depressed and socially anxious adolescents (Van Zalk, Kerr, Branje, Stattin, \& Meeus, 2010; Van Zalk, Van Zalk, Kerr, \& Stattin, 2011). Thus, there is support for a greater likelihood of friendship formation among children with similar levels of internalizing problems.

A recent study found that selection similarity in internalizing problems may also be the result of fewer overall opportunities for friendship formation due to active withdrawal. Schaefer, Kornienko, and Fox (2011) argued that nondepressed individuals prefer nondepressed friends because they provide the greatest rewards. Among depressed individuals, friendships with nondepressed peers are, according to Schaefer et al. (2011), also the most rewarding and hence most desirable. Depressed adolescents, however, often withdraw from friendships and become marginalized in the 
broader friendship network, which excludes them from the normative network processes that help build and sustain friendships.

Influence processes offer an alternative explanation for why adolescents are similar to their peers in internalizing symptoms. Mercer and DeRosier (2010) reported that friends' anxiety, depression, and loneliness influenced children's own internalizing problems (see also Van Zalk et al., 2010, 2011). They argued that anxious and depressive feelings and thoughts may increase through reinforcement of negative cues in interactions with friends with internalizing symptoms. In sum, adolescents tend to select peers with similar levels of internalizing problems and may increase each other's internalizing problems as relationships endure (see also Cheadle \& Goosby, 2012).

In an exemplary study in this issue, Giletta, Burk, Scholte, Engels, and Prinstein (2013) examined processes of direct and indirect socialization. They investigated whether nonsuicidal self-injury by adolescents may result not only from such behavior by their friends but also from other characteristics of friends that promote risky environments for adolescent development. They found evidence for such indirect socialization. First, the depressive symptoms of their friends predicted changes in adolescents' nonsuicidal self-injury. Second, the impulsivity of their friends predicted changes in nonsuicidal self-injury among boys. Thus, the context that friends provide, in terms of behaviors and attitudes, may influence adolescents because they may offer the conditions for development of certain behaviors by acceding to the adolescents' behavior or exacerbating their individual predisposing characteristics. The use of multivariate network-behavior dynamics models allows researchers to examine such cross-dimensional influence processes.

\section{Substance Use}

In another exemplary study in this issue, Mathys, Burk, and Cillessen (2013) used a multivariate network-behavior dynamics model to compare selection and influence mechanisms for alcohol use, marijuana use, and smoking simultaneously. It turned out that selection played a greater role than influence for explaining similarity in the smoking behavior of friends in middle and late adolescence (see also Kiuru et al., 2010; Mercken, Steglich, Knibbe, \& De Vries, 2012). This finding is consistent with the idea that tobacco use is addictive and that once adolescents have started smoking, social influence may become less important for smoking progression. Spatial segregation of smokers may provide an explanation for the selection effect: society separates smokers from nonsmokers (referring to smoking-designated areas), creating an opportunity structure in which smokers are more likely to establish new contacts with other smokers and then affiliate with each other. Influence processes, however, seem to play a role in tobacco use in early adolescence (Huisman \& Bruggeman, 2012; Mercken, Steglich, Sinclair, Holliday, \& Moore, 2012; Steglich, Sinclair, Holliday, \& Moore, 2012).

Mathys et al. (2013) showed that neither selection nor influence played a role in explaining similarity in marijuana use (but see also De la Haye, Green, et al., 2013). Finally, Mathys et al. (2013) found that only influence played a significant role in explaining similarity in drinking behavior (see also Osgood et al., 2013).

\section{RECENT EMPIRICAL ADVANCES}

\section{Deselection}

The main argument for examining selection and influence jointly has been that both processes explain similarity between friends. Deselection offers a third explanation for similarity; if friends deselect those who are dissimilar to them, the remaining friends will be more similar to each other than nonfriends. Both Van Zalk et al. (2010) and Kiuru, Burk, Laursen, Nurmi, and Salmela-Aro (2012) examined selection and deselection processes in relation to adolescent depressive symptoms. Based on the theory of social corrosion (Coyne, 1976), they hypothesized that individuals prone to depressive symptoms lack the social skills necessary to provide support and closeness, which, in turn, triggers dissatisfaction and even deselection by the nondepressive dyadic partner in the relationship, and therefore increases the chances of a close relationship ending. In contrast, they hypothesized that interactions between two depressive friends are characterized by mutual feelings of understanding and high self-disclosure, which seem to increase closeness and intimacy between these friends (Rose, 2002). This, in turn, is suggested to lead to fewer endings of relationships between two depressed friends. The findings of Kiuru et al. (2012) were in line with these ideas, whereas Van Zalk et al. (2010) found that similarity in depression fostered tie creation but not tie dissolution.

The study by Van Workum, Scholte, Cillessen, Lodder, and Giletta (2013) further confirms the 
importance of examining deselection to understand the role of emotional development in network dynamics. The authors suggest that adolescents who have friends who are unhappier than themselves may be influenced by these friends' moods, which may induce negative feelings. These negative feelings may trigger dissatisfaction in the friendship and, therefore, increase the chances of the friendship ending. Their findings show that lower similarity predicted higher deselection.

These studies open up future opportunities for empirical examination of mediating mechanisms that explain deselection. Thus, rather than being the mere opposite of selection, deselection processes may offer unique insights into the mechanisms that explain how relationships are maintained and provide an alternative explanation for why adolescents may have friends who are similar to them.

\section{Moderating Effects in Influence Processes}

Examining moderating effects in peer influence processes also adds to our understanding of dynamics in the peer context. In theory, influence effects can be moderated by characteristics of individuals (e.g., impulsivity), peers (e.g., status), the dyadic relationship (e.g., friendship quality), and the context (e.g., density of the network) (Brechwald \& Prinstein, 2011).

Moderating effects of individual characteristics have been tested in some studies. It was found that boys are more sensitive to the influence of delinquent friends (Burk, Steglich, \& Snijders, 2007) and that girls are more sensitive to the influence of depressed friends (Van Zalk et al., 2010). Impulsive youth were found to be more susceptible to the influence of friends' alcohol use (Rabaglietti, Burk, \& Giletta, 2012). In this issue, Molano et al. (2013) found that adolescents with high levels of hostile attribution bias were found to be more inclined to adopt the aggression of their peers. Youth with psychopathic traits as well as high-status youth were found to be less susceptible to the influence of friends' delinquency (Kerr, Van Zalk, \& Stattin, 2012). Overall, it appears that there might be individual risk factors that accentuate risk of peer influence.

It is also relevant to examine the relative extent of influence. Kerr et al. (2012) were the first to test using SIENA whether some adolescents were more influential than others. They found that peers with psychopathic traits were more influential.

Overall, there is no consistent evidence in social network models that mutual friends have a stronger influence than unilateral friends (see for two exceptions Burk et al., 2007; Ojanen, Sijtsema, Hawley, \& Little, 2010). Other measures for closeness or intimacy of friendships have not been included in actor-based models of behavior dynamics so far.

Rambaran, Dijkstra, and Stark (2013) and DeLay, Laursen, Kiuru, Nurmi, and Salmela-Aro (2013) are among the first to have tested whether influence effects depend on contextual characteristics. Rambaran et al. (2013) found that influence effects on risk attitudes were stronger in a context where status was positively correlated with risk attitudes. DeLay et al. (2013) found that processes of selecting new friends based on similarity were stronger for adolescents in groups with a low rate of smoking, whereas processes of dropping friends based on dissimilarity were stronger for adolescents in groups with a high rate of smoking.

\section{Moderating Effects in Selection Processes}

Certain individual, relational, or contextual factors may make selection of similar friends, or friends with particular attributes, more or less appealing. This may have important implications for whether youth find themselves in risky versus protective peer contexts. For that reason, it is important to look at moderation in selection processes.

De la Haye, Robins, Mohr, and Wilson (2011) provided evidence for a sex difference in the selection similarity effect for weight status and Kiuru et al. (2010) for a sex difference in the selection similarity effect for alcohol use. Burk et al. (2012) found some evidence for sex differences in peer selection based on alcohol intoxication. Their results suggest that drinking behaviors were initially a more important selection criterion for friendships of early adolescent males, whereas females were more likely to select peer affiliates with similar drinking behaviors in late adolescence.

In this issue, Simpkins et al. (2013) found that selection of friends based on BMI and physical activity was moderated by adolescents' sex, friends' popularity, and friendship reciprocity. Adolescents with higher BMIs were more likely to be selected as friends within same-sex friendships than other-sex friendships, and selection similarity based on BMI was stronger when selecting female friends than male friends. They also found that selection based on physical activity was less important when friends were popular than when friends were less popular. Furthermore, being similar in terms of BMI was more important for unreciprocated friendships than reciprocated ones. 
Mathys et al. (2013) found that popular children sought friends with higher alcohol use. Rulison et al. (2013) found that girls were more likely to select physically aggressive friends than boys and that peer-rejected youth were less likely to select physically aggressive friends.

As far as we know, these are the first studies to examine moderation in selection processes. Future research on network-behavior dynamics needs to incorporate such interactions too.

\section{Time Heterogeneity Effects}

One aspect of network-behavior dynamics that merits our attention is time heterogeneity, referring to differences in the occurrence of selection and influence processes over time. In an exemplary study, Burk et al. (2012) examined when selection and influence effects of alcohol intoxication emerge, peak, and desist from ages 10 to 18 . Their findings indicated that similarity in same-age friends' drinking behaviors emerged in early adolescence, peaked in middle adolescence, and decreased throughout late adolescence. Several contributions to this issue also looked at time heterogeneity and found no differences over time (De la Haye, Robins, Mohr, \& Wilson, 2013; Giletta et al., 2013; Van Workum et al., 2013). It should be noted that Burk et al. (2012) looked at time heterogeneity during an eight-year time window, whereas the studies in this special issue had a time frame of two years at the most.

\section{Initiation of Behavior}

Newly developed stochastic actor-based diffusion models were applied in two studies reported in this issue (De la Haye, Green, et al., 2013; Light et al., 2013). These models estimate effects on the rate of initiation of behavior. Light et al. (2013) linked exposure to friends who had already begun drinking to the initiation of drinking. They found that peer exposure had a strong effect on initiation of alcohol use and proposed that such exposure supplies information about socially attractive events like parties with popular peers as well as a social context in which peers model and approve of such behavior. Thus, Light et al. (2013) found an increased rate of onset to first alcohol use in early adolescence based on exposure to already-onset peers, whereas Burk et al. (2012) found no effect on acceleration. Studies are needed that compare peer effects on initiation versus acceleration across adolescence to find out whether or not different stages of a behavior (referring to the first time a behavior is tried versus adopting the behavior regularly) are predicted by different factors.

\section{Mediation Effects}

Longitudinal social network models also allow researchers to examine mediation processes that help explain why adolescents change their network or their behavior, such as the cognitive or behavioral processes that underpin the phenomenon of youth adopting the behaviors of their friends (see also Van Zalk-Selfhout, Kerr, Branje, Stattin, \& Meeus, 2010). De la Haye, Robins, Mohr, and Wilson (2013) tested mediation effects proposed in social cognition theories, but found none. It turned out that changes to adolescents' beliefs about junk food did not appear to be the mechanisms underpinning influence from their friends. Interestingly, intake of junk food predicted changes in cognitions, so that greater intake predicted stronger intentions to consume junk food, more positive attitudes toward these foods, and stronger beliefs that friends regularly consumed these foods. These results are in line with theories of self-perception (Bem, 1972) and mindless eating (Wansink \& Sobal, 2007).

\section{Negative Ties}

Traditionally, the focus of social network analysis has been on relationships with a positive meaning, such as friendship. A recent study by Huitsing et al. (2012) showed that negative tie networks can be modeled and that they are meaningfully related to positive tie networks. They found that there were positive ties between individuals who were structurally equivalent in the negative tie network, in line with balance theory. In this issue, Berger and Dijkstra (2013) used a multiple network approach and focused on how popularity and friendships affect the development of dislike relationships, so-called antipathies, over time. They showed that dissimilarity in popularity shapes antipathies and that friends tend to agree upon which peers to dislike over time.

\section{CONCLUSION}

Research on network-behavior dynamics has expanded in recent years, making important conceptual, methodological, and empirical contributions to an important area of study. In this issue, great strides have been made to move beyond merely demonstrating that selection and influence 
processes coevolve. These studies have shown that selection and influence processes occur within a social system that is dynamic and that understanding the broader properties of this system (structural network characteristics, selection processes, behavioral tendencies, and influence processes) is essential for understanding how networks and behaviors develop.

An important future research aim is to examine how interventions aimed at reducing negative behaviors or promoting positive behaviors are influenced by network-behavior dynamics (Steglich et al., 2012). Although much work is still needed, this issue has provided evidence on moderating effects, initiation of behaviors, time heterogeneity, mediation effects, and negative ties. This new evidence is likely to advance theories of social development and peer relationships and provide useful insights for a range of practical applications such as prevention efforts and adolescent policy.

\section{REFERENCES}

Note. The asterisks denote that SIENA was used.

Bandura, A. (1977). Social learning theory. Englewood Cliffs, NJ: Prentice Hall.

Bearman, P. (1997). Generalized exchange. American Journal of Sociology, 102, 1383-1415.

Bem, D. J. (1972). Self-perception theory. Advances in Experimental Social Psychology, 6, 1-62.

*Berger, C., \& Dijkstra, J. K. (2013). Competition, envy, or snobbism? How popularity and friendships shape antipathy networks of adolescents. Journal of Research on Adolescence, 23, 586-595. doi:10.1111/jora.12048

Blau, P. M. (1977). A macrosociological theory of social structure. American Journal of Sociology, 83, 26-54.

Brechwald, W. A., \& Prinstein, M. J. (2011). Beyond homophily: A decade of advances in understanding peer influence processes. Journal of Research on Adolescence, 21, 166-179.

Burgess, R. L., \& Akers, R. L. (1966). A differential association-reinforcement theory of criminal behavior. Social Problems, 14, 128-147.

*Burk, W. J., Steglich, C. E. G., \& Snijders, T. A. B. (2007). Beyond dyadic interdependence: Actor-oriented models for co-evolving social networks and individual behaviors. International Journal of Behavioral Development, 31, 397-404.

*Burk, W. J., Van der Vorst, H., Kerr, M., \& Stattin, H. (2012). Alcohol use and friendship dynamics: Selection and socialization in early-, middle-, and late-adolescent peer networks. Journal of Studies on Alcohol and Drugs, 73, 89-98.

Byrne, D. E. (1971). The attraction paradigm. New York, NY: Academic Press.
Cartwright, D., \& Harary, F. (1956). Structural balance: A generalization of Heider's theory. Psychological Review, 63, 277-293.

${ }^{*}$ Cheadle, J. E., \& Goosby, B. J. (2012). The small-school friendship dynamics of adolescent depressive symptoms. Society and Mental Health, 2, 99-119.

${ }^{*}$ Cheadle, J. E., \& Schwadek, P. (2012). The 'friendship dynamics of religion' or the 'religious dynamics of friendship'? A social network analysis of adolescents who attend small schools. Social Science Research, 41, 1198-1212.

Cohen, J. M. (1977). Sources of peer group homogeneity. Sociology of Education, 50, 227-241.

Coyne, J. C. (1976). Depression and the response of others. Journal of Abnormal Psychology, 85, 186-193.

*Dahl, V., \& Van Zalk, M. (2014). Peer networks and the development of illegal political behavior among adolescents. Journal of Research on Adolescence. doi:10.1111/ jora. 12072

Davis, J. A. (1970). Clustering and hierarchy in interpersonal relations: Testing two graph theoretical models on 742 sociomatrices. American Sociological Review, 35, 843-851.

*De la Haye, K., Green, H. D., Kennedy, D., Pollard, M., \& Tucker, J. (2013). Selection and influence mechanisms associated with marijuana initiation and use in adolescent friendship networks. Journal of Research on Adolescence, 23, 474-486. doi:10.1111/jora.12018

*De la Haye, K., Robins, G., Mohr, P., \& Wilson, C. (2011). Homophily and contagion as explanations for weight similarities among adolescent friends. Journal of Adolescent Health, 49, 421-427.

*De la Haye, K., Robins, G., Mohr, P., \& Wilson, C. (2013). Adolescents' intake of junk food: Processes and mechanisms driving consumption similarities among friends. Journal of Research on Adolescence, 23, 524-536. doi:10.1111/jora.12045

*DeLay, D., Laursen, B., Kiuru, N., Nurmi, J.-E., \& Salmela-Aro, K. (2013). Selecting and retaining friends on the basis of cigarette smoking similarity. Journal of Research on Adolescence, 23, 464-473. doi:10.1111/jora. 12017

*Dijkstra, J. K., Berger, C., \& Lindenberg, S. (2011). Do physical and relational aggression explain adolescents' friendship selection? The competing roles of network characteristics, gender, and social status. Aggressive Behavior, 37, 417-429.

*Dijkstra, J. K., Cillessen, A. H. N., \& Borch, C. (2013). Popularity and adolescent friendship networks: Selection and influence dynamics. Developmental Psychology, 49, 1242-1252. doi:10.1037/a0030098

*Dijkstra, J. K., Gest, S. D., Lindenberg, S., Veenstra, R., \& Cillessen, A. H. N. (2012). Testing three explanations of the emergence of weapon carrying in peer context. The role of aggression, victimization, and the social network. Journal of Adolescent Health, 48, 371-376.

*Dijkstra, J. K., Lindenberg, S., Veenstra, R., Steglich, C., Isaacs, J., Card, N. A., \& Hodges, E. V. E. (2010). Influ- 
ence and selection processes in weapon carrying during adolescence: The roles of status, aggression, and vulnerability. Criminology, 48, 187-220.

Durkheim, E. (1897). Suicide: A study in sociology. London, UK: Routledge.

Feld, S. L. (1982). Social structural determinants of similarity among associates. American Sociological Review, 47, 797-801.

Friedkin, N. E. (1998). A structural theory of social influence. Cambridge, UK: Cambridge University Press.

*Giletta, M., Burk, W., Scholte, R., Engels, R., \& Prinstein, M. J. (2013). Direct and indirect peer socialization of adolescent nonsuicidal self-injury. Journal of Research on Adolescence, 23, 450-463. doi:10.1111/jora. 12036

Hallinan, M. T. (1980). Patterns of cliquing among youth. In H. C. Foot, A. J. Chapman, \& J. R. Smith (Eds.), Friendship and social relations in children (pp. 321-342). Chichester, UK: Wiley.

Holland, P. W., \& Leinhardt, S. (1977). Dynamic-model for social networks. Journal of Mathematical Sociology, 5, 5-20.

*Huisman, C., \& Bruggeman, J. (2012). The social network, socioeconomic background, and school type of adolescent smokers. International Journal of Behavioral Development, 36, 329-338.

Huitsing, G., Van Duijn, M. A. J., Snijders, T. A. B., Wang, P., Sainio, M., Salmivalli, C., \& Veenstra, R. (2012). Univariate and multivariate models of positive and negative networks: Liking, disliking, and bullyvictim relationships social networks. Social Networks, 34, 645-657.

*Kerr, M., Van Zalk, M., \& Stattin, H. (2012). Psychopathic traits moderate peer influence on adolescent delinquency. Journal of Child Psychology and Psychiatry, $53,826-836$.

*Kiuru, N., Burk, W. J., Laursen, B., Nurmi, J.-E., \& Salmela-Aro, K. (2012). Is depression contagious? A test of alternative peer socialization mechanisms of depressive symptoms in adolescent peer networks. Journal of Adolescent Health, 50, 250-255.

*Kiuru, N., Burk, W., Laursen, B., Salmela-Aro, K., \& Nurmi, J.-E. (2010). Pressure to drink but not to smoke: Disentangling selection and socialization in adolescent peer networks and peer groups. Journal of Adolescence, 33, 801-812.

*Knecht, A., Snijders, T. A. B., Baerveldt, C., Steglich, C. E. G., \& Raub, W. (2010). Friendship and delinquency: Selection and influence processes in early adolescence. Social Development, 19, 494-514.

Lazarsfeld, P. F., \& Merton, R. K. (1954). Friendship as social process: A substantive and methodological analysis. In M. Berger, T. Abel, \& C. H. Page (Eds.), Freedom and control in modern society (pp. 18-66). New York, NY: Van Nostrand.

*Light, J. M., Greenan, C. C., Rusby, J. C., Nies, K. M., \& Snijders, T. A. B. (2013). Onset to first alcohol use in early adolescence: A network diffusion model.
Journal of Research on Adolescence, 23, 487-499. doi:10. 1111 /jora.12064

*Logis, H., Rodkin, P. C., Gest, S. D., \& Ahn, H.-J. (2013). Popularity as an organizing factor of preadolescent friendship networks: Beyond prosocial and aggressive behavior. Journal of Research on Adolescence, 23, 413-423. doi:10.1111/jora.12033

*Mathys, C., Burk, W. J., \& Cillessen, A. H. N. (2013). Popularity as moderator of peer selection and socialization of adolescent alcohol, marijuana, and tobacco use. Journal of Research on Adolescence, 23, 513-523. doi:10.1111/jora.12031

McPherson, M., Smith-Lovin, L., \& Cook, J. M. (2001). Birds of a feather: Homophily in social networks. Annual Review of Sociology, 27, 415-444.

*Mercer, S. H., \& DeRosier, M. (2010). Selection and socialization of internalizing problems in middle childhood. Journal of Social and Clinical Psychology, 29, 1032-1059.

*Mercken, L., Steglich, C., Knibbe, R., \& De Vries, H. (2012). Dynamics of friendship networks and alcohol use in early and mid-adolescence. Journal of Studies on Alcohol and Drugs, 73, 99-110.

*Mercken, L., Steglich, C., Sinclair, P., Holliday, J., \& Moore, L. (2012). A longitudinal social network analysis of peer influence, peer selection, and smoking behavior among adolescents in British schools. Health Psychology, 31, 450-459.

Merton, R. K. (1968). The Matthew effect in science. Science, 159, 56-63.

*Molano, A., Jones, S., Brown, J., \& Aber, J. L. (2013). Selection and socialization of aggressive and prosocial behavior: The moderating role of social-cognitive processes. Journal of Research on Adolescence, 23, 424-436. doi:10.1111/jora.12034

*Ojanen, T., Sijtsema, J. J., Hawley, P. H., \& Little, T. D. (2010). Intrinsic and extrinsic motivation in early adolescents' friendship development: Friendship selection, influence, and prospective friendship quality. Journal of Adolescence, 33, 837-851.

Osgood, D. W., \& Anderson, A. L. (2004). Unstructured socializing and rates of delinquency. Criminology, 42, 519-549.

*Osgood, D. W., Ragan, D. T., Wallace, L., Gest, S. D., Feinberg, M. E., \& Moody, J. (2013). Peers and the emergence of alcohol use: Influence and selection processes in adolescent friendship networks. Journal of Research on Adolescence, 23, 500-512. doi:10.1111/jora. 12059

*Rabaglietti, E., Burk, W. J., \& Giletta, M. (2012). Regulatory self-efficacy as a moderator of peer socialization relating to Italian adolescents' alcohol intoxication. Social Development, 21, 522-536.

*Rambaran, J. A., Dijkstra, J. K., \& Stark, T. H. (2013). Status-based influence processes: The role of norm salience in contagion of adolescent risk attitudes. Journal of Research on Adolescence, 23, 574-585. doi:10.1111/jora. 12032 
Rose, A. J. (2002). Co-rumination in the friendships of girls and boys. Child Development, 73, 1830-1843.

*Rulison, K. L., Gest, S. D., \& Loken, E. (2013). Dynamic social networks and physical aggression: The moderating role of gender and social status among peers. Journal of Research on Adolescence, 23, 437-449. doi:10.1111/jora.12044

*Schaefer, D. R., Kornienko, O., \& Fox, A. M. (2011). Misery does not love company: Network selection mechanisms and depression homophily. American Sociological Review, 76, 764-785.

*Sentse, M., Dijkstra, J. K., Salmivalli, C., \& Cillessen, A. H. N. (2013). The dynamics of friendships and victimization in adolescence: A longitudinal social network perspective. Aggressive Behavior, 39, 229-238.

*Sijtsema, J. J., Ojanen, T., Veenstra, R., Lindenberg, S., Hawley, P. H., \& Little, T. D. (2010). Forms and functions of aggression in adolescent friendship selection and influence: A longitudinal social network analysis. Social Development, 19, 515-534.

*Sijtsema, J. J., Rambaran, J. A., \& Ojanen, T. J. (2013). Overt and relational victimization and adolescent friendships: Selection, de-selection, and social influence. Social Influence, 8, 177-195.

*Simpkins, S. D., Schaefer, D. R., Price, C. D., \& Vest, A. E. (2013). Adolescent friendships, BMI, and physical activity: Untangling selection and influence through longitudinal social network analysis. Journal of Research on Adolescence, 23, 537-549. doi:10.1111/j.1532-7795. 2012.00836.x

Snijders, T., Steglich, C., \& Schweinberger, M. (2007). Modeling the coevolution of networks and behavior. In K. Van Montfort, J. Oud, \& A. Satorra (Eds.), Longitudinal models in the behavioral and related sciences (pp. 41-71). Mahwah, NJ: Lawrence Erlbaum.

Snijders, T. A. B., Van de Bunt, G. G., \& Steglich, C. E. G. (2010). Introduction to stochastic actor-based models for network dynamics. Social Networks, 32, 44-60.

*Steglich, C., Sinclair, P., Holliday, J., \& Moore, L. (2012). Actor-based analysis of peer influence in $A$ Stop Smoking in Schools Trial (ASSIST). Social Networks, 34, 359-369.

*Steglich, C., Snijders, T. A. B., \& Pearson, M. (2010). Dynamic networks and behavior: Separating selection from influence. Sociological Methodology, 41, 329-393.

*Svensson, Y., Burk, W. J., Stattin, H., \& Kerr, M. (2012). Peer selection and influence of delinquent behavior of immigrant and nonimmigrant youths: Does context matter? International Journal of Behavioral Development, 36, 178-185.

*Van Workum, N., Scholte, R. H. J., Cillessen, A. H. N., Lodder, G. M. A., \& Giletta, M. (2013). Selection, deselection, and socialization processes of happiness in adolescent friendship networks. Journal of Research on Adolescence, 23, 563-573. doi:10.1111/jora.12035

*Van Zalk, M. H. W., Kerr, M., Branje, S. J. T., Stattin, H., \& Meeus, W. H. J. (2010). It takes three: Selection, influence, and de-selection processes of depression in adolescent friendship networks. Developmental Psychology, 46, 927-938.

*Van Zalk, M. H. W., Kerr, M., Van Zalk, N., \& Stattin, H. (2013). Xenophobia and tolerance toward immigrants in adolescence: Cross-influence processes within friendships. Journal of Abnormal Child Psychology, 41, 627-639.

*Van Zalk, N., Van Zalk, M., Kerr, M., \& Stattin, H. (2011). Social anxiety as a basis for friendship selection and socialization in adolescents' social networks. Journal of Personality, 79, 499-526.

*Van Zalk-Selfhout, M., Kerr, M., Branje, S., Stattin, H., \& Meeus, W. (2010). Peer contagion and adolescent depression: The role of failure anticipation. Journal of Clinical Child and Adolescent Psychology, 39, 837-848.

Veenstra, R., \& Dijkstra, J. K. (2011). Transformations in adolescent peer networks. In B. Laursen \& W. A. Collins (Eds.), Relationship pathways: From adolescence to young adulthood (pp. 135-154). Los Angeles, CA: Sage.

Veenstra, R., \& Steglich, C. (2012). Actor-based model for network and behavior dynamics. In B. Laursen, T. D. Little, \& N. A. Card (Eds.), Handbook of developmental research methods (pp. 598-618). New York, NY: Guilford Press.

Wansink, B., \& Sobal, J. (2007). Mindless eating. Environment and Behavior, 39, 106-123.

Warr, M. (2002). Companions in crime: The social aspects of criminal conduct. Cambridge, UK: Cambridge University Press.

*Weerman, F. (2011). Delinquent peers in context: A longitudinal network analysis of selection and influence effects. Criminology, 49, 253-286.

Zajonc, R. B. (1968). Attitudinal effects of mere exposure. Journal of Personality and Social Psychology, 9, 2P2, 1-27. 
This document is a scanned copy of a printed document. No warranty is given about the accuracy of the copy. Users should refer to the original published version of the material. 